\title{
Empoderar el Emprendimiento Femenino Universitario
}

\author{
Catherine Krauss Delorme ${ }^{1 *}$, Adriana Bonomo Odizzio ${ }^{1}$, Roberto Volfovicz León ${ }^{1}$
}

\begin{abstract}
In Latin America, university entrepreneurial education must adapt to the new context of the 4th Revolution and encourage women's entrepreneurial intention. The objective was to analyze the challenge of universities in the promotion of women's EIs so that they have a positive impact, and undertake as a career choice, understood as an entrepreneurial activity or the creation of a new business. An analysis based on structural equations was performed to measure its impact on entrepreneurial intention. The most relevant result: Personal Attitude, Perceived Internal Control, Family and University Contexts have a significant and direct effect on the entrepreneurial intention of university students.
\end{abstract}

Keywords : Entrepreneurial intention; women entrepreneurship; entrepreneurship education; entrepreneurial attitude

Resumen: En América Latina la educación emprendedora universitaria debe adaptarse al nuevo contexto de la 4ta Revolución y fomentar la intención emprendedora (IE) femenina. El objetivo planteado fue analizar el desafío de las universidades en el fomento de las IE femeninas para que tengan un impacto positivo, y emprendan como elección de carrera, entendido como una actividad emprendedora o la creación de un nuevo negocio. Se realizó un análisis basado en ecuaciones estructurales para medir su impacto sobre la Intención Emprendedora. El resultado más relevante: la Actitud Personal, el Control Interno Percibido, los Contextos Familiar y Universitario tienen un efecto significativo y directo sobre la Intención Emprendedora de las estudiantes universitarias.

Palabras clave: Intención emprendedora; emprendimiento femenino; educación emprendedora; actitud emprendedora.

Submitted: January $2^{\text {nd }}, 2020 /$ Approved: July $24^{\text {th }}, 2020$

\section{Introducción}

El estudio busca analizar el desafío que tienen las universidades para empoderar a sus estudiantes y fomentar el emprendimiento femenino. La 4ta Revolución industrial trae aparejado oportunidades sin precedentes, así como la necesidad del desarrollo de las capacidades y habilidades como la creatividad, la empatía y el ingenio (Din, Anuar, y Usman, 2016; Krauss, Bonomo y Volfovicz, 2018(a); Welsh, Tullar y Nemati, 2016). Es crítico y fundamental llegar a la contribución igualitaria de mujeres y hombres para hacer frente a los cambios acelerados que se producen y lograr un futuro más próspero y humano céntrico. En el área de Inteligencia Artificial, únicamente un 22\% de los profesionales son mujeres, indicando un límite importante a nivel de género a la capacidad innovadora e inclusiva y una alta pérdida de oportunidades (World Economics Forum, 2018).

Según el Instituto McKinsey Global Institute (2015) se podrían agregar \$ 12 billones al PIB (Producto Interno Bruto) mundial para 2025 mediante el avance de la igualdad de las mujeres. Para lograrlo, los sectores públicos, privados y sociales deberán cerrar las brechas de género en el trabajo y en la sociedad mediante: incentivos financieros; tecnología e infraestructura; creación de oportunidades económicas y desarrollo de capacidades a través de la educación.

Los indicadores para Uruguay, del Global Entrepreneurship Monitor GEM (2016/2017), lo ubican en el nivel de desarrollo "Transición Eficiencia-Innovación”, la TEA (Actividad Emprendedora Total) femenina para Uruguay es de $9,9 \%$, por necesidad son $35,3 \%$, y por oportunidad $63,5 \%$ y la intención emprendedora femenina es 25,5\%. Entre los factores que afectan el desarrollo empresarial de la mujer en Uruguay, se observa que sólo el 35\% de las mujeres tiene acceso a cargos directivos en la empresa MiPymes (MIEM, 2017). De acuerdo con el GEM, en Uruguay las mayores barreras percibidas por los emprendedores nacientes son para las mujeres el área financiera y para los hombres, la comercial. (Veiga, Chaquiriand \& Bartesaghi, 2017).

El objetivo de esta investigación es analizar el desafío de las universidades en las intenciones emprendedoras femeninas para que al empoderarlas tengan un impacto positivo sobre la intención de emprender como elección de carrera. Esta investigación se estructura de la siguiente forma, primero la introducción y objetivos; segundo el marco teórico; tercero, la metodología aplicada; cuarto, el desarrollo de la investigación donde se señalan los resultados obtenidos y finalmente conclusiones y recomendaciones.

\section{Marco Teórico}

Analizando a las mujeres que han emprendido, se ha podido constatar que han desarrollado competencias relacionadas con el autoconocimiento y el manejo de sus propias emociones, pero, presentan carencias en el liderazgo, en la gestión de las personas y en lograr sus objetivos (Ortiz García \& Olaz Capitán, 2018).

En el empoderamiento del emprendimiento femenino existen factores que afectan su vida empresarial, los motivacionales de pull y push, balance y emocionales. Los que motivan internamente a las

1) Centro Ithaka de Emprendimiento e Innovación, Departamento de Administración y Finanzas, Universidad Católica del Uruguay, Montevideo, Uruguay.

*Corresponding author: ckrauss@ucu.edu.uy 
mujeres a emprender y están más relacionados con la autorrealización, son los llamados pull. Los factores externos, push, están vinculados, por ejemplo, a los incentivos que reciben a través de un despido, a la insatisfacción laboral, etc. Ambos factores ( pull y push) están concentrados especialmente en el deseo de independencia, en la motivación financiera, y en los temas relacionados a la familia y el negocio (Özsungur, 2019). Los factores de balance están motivados por el rápido desarrollo de la tecnología, y su falta de tiempo causando una brecha entre la familia y el emprendimiento; lo que hace que la flexibilidad en el negocio sea un factor de mucha influencia en la decisión de emprender (Stevenson, 1986). Los factores emocionales y cognitivos como el compromiso con el trabajo, la lealtad, la solidaridad, la necesidad de redes, el soporte familiar y personal, tienen un impacto significativo en el emprendimiento femenino (Özsungur, 2019).

Varios estudios hallaron una relación positiva entre la intención emprender y la formación en los negocios, así como la habilidad de identificar oportunidades, entendiendo la acción emprendedora como un comportamiento orientado a objetivos, e influenciado directamente por la IE (Krueger, Reilly \& Carsrud, 2000; Nicolaou, Shane, Cherkas \& Spector, 2009).

Para esta investigación se utilizó el marco de referencia de la Teoría del Comportamiento Planificado (TPB- Theory of Planned Behavior) de Ajzen (1991), que sirve para pronosticar la intención de emprender como un indicador del esfuerzo que realizarán para ser emprendedores, en este caso las estudiantes. Este comportamiento se basa en tres factores:-control interno percibido, las normas subjetivas y sociales; y la actitud personal (Krueger et al., 2000; Liñán \& Chen, 2009; Van Gelderen, Kautonen, \& Fink, 2015). Comenzando con el control percibido, se considera la IE como una de las variables importantes para mostrar el efecto del locus de control en el reconocimiento de oportunidades de los aspirantes a emprendedores existiendo dos diferentes tipos de percepción de locus de control a nivel individual: interno y externo (Fayolle \& Liñan, 2014). El locus de control es importante por la forma en que percibe la persona el entorno. Los individuos que poseen un alto locus de control interno tienen la percepción que no pueden influenciar los eventos exteriores afectando en la decisión de emprender e impacta en la importancia de su autoeficacia (Asante \& Affum-Osei, 2019; Esfandiar, Sharifi-Tehrani, Pratt, \& Altinay, 2019).

Según el modelo del TBP, las normas subjetivas y sociales afectan en la IE, siendo las acciones emprendedoras más valoradas en algunas culturas que en otras (Bogatyreva, Edelman, Manolova, \& Osiyevskyy, 2019), pudiendo esto, influir en la IE y la futura carrera empresarial (Newbery, Lean, Moizer, \& Haddoud, 2018).

Asimismo, el estereotipo de emprendimiento negativo en las mujeres puede ser muy fuerte y de mucho peso a la hora de emprender, tanto en países en desarrollo (Roomi, Rehman, \& Henry, 2018) como en países desarrollados (Joensuu, Viljamaa, \& Varamaki, 2013). A pesar de los valores culturales y religiosos, se ha constatado los beneficios de la educación en estudiantes universitarias femenina (Azis, Maulana Haeruddin, \& Azis, 2018). Asimismo, se ha investigado que existe un desajuste entre la IE de los estudiantes mientras realizan su carrera y el hecho de emprender; ya que lanzar una startup antes de la graduación requiere muchas actividades que podrían afectar el resultado, siendo la IE medida, mayor en el período después de la graduación (Bogatyreva et al., 2019). Los estereotipos y las percepciones sociales de género en una determinada cultura que consideren en sus normas sociales como no apropiado el emprendimiento femenino o el que sus emprendimientos sean asimilables a su unidad doméstica, carencia de formación y gestión financiera, provocan que los emprendimientos de las mujeres sean en rubros menos rentables, más pequeños y menos orientados al crecimiento que los manejados por hombre, como se pudo visualizar en el caso Chile y de los Emiratos Árabes (Naranjo, 2019; Van Ewijk \& Belghiti-Mahut, 2019).

Con respecto a la actitud emprendedora, la influencia de tener personas emprendedoras como modelo de roles, hace que sus actitudes y la autoeficacia emprendedora sean más efectivas para fortalecer la IE de los estudiantes universitarios (Nowinski \& Haddoud, 2019). En particular, tener padres emprendedores exitosos, especialmente en las empresas familiares, influencia positivamente contribuyendo tanto a la intención como a la actividad emprendedora (Criaco, Sieger, Wennberg, Chirico, \& Minola, 2017).

En este trabajo se busca analizar si las estudiantes manifiestan actitud y control percibido interno hacia la IE, (Ajzen, 1991; Liñán \& Chen, 2009; Marín Rives, Rubio Bañón, \& Sánchez-Mora, 2015) para convertirse en empresarias. A continuación, se presenta la figura 1 con las relaciones y las diferentes hipótesis del trabajo.

Figura 1. Modelo de investigación. Elaboración propia basado en (Ajzen, 1991; Sieger, Fueglistaller, \& Zellweger, 2014)

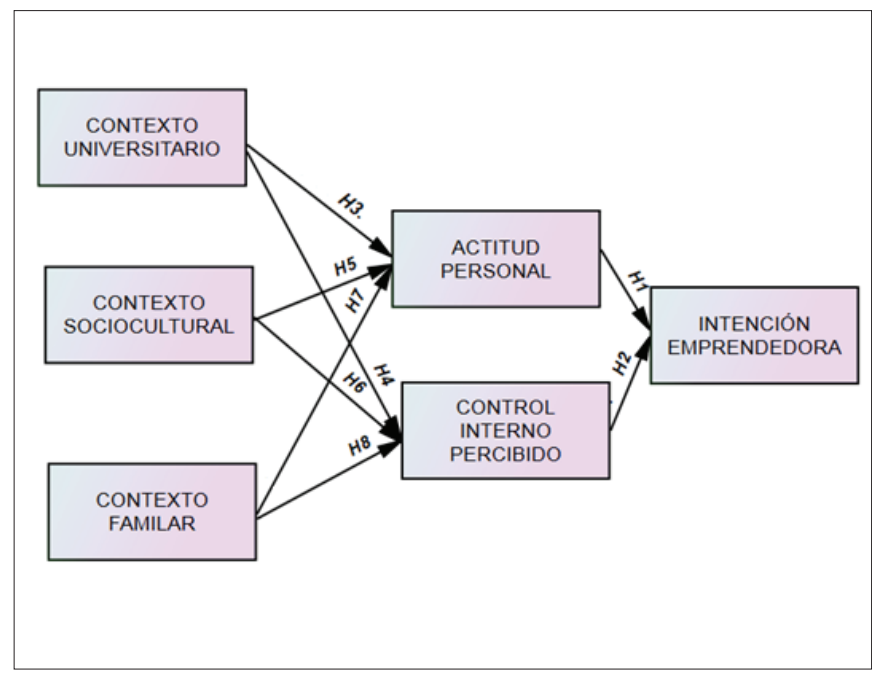

H1- La actitud personal de la estudiante tiene un efecto positivo en la IE como carrera al finalizar sus estudios.

El reconocimiento de oportunidades para emprendedores nacientes, y el efecto del locus de control ( Fayolle \& Liñan, 2014), es clave para el proceso emprendedor (Asante \& Affum-Osei, 2019); un locus de control interno alto genera potencialmente que desarrolle su propio negocio. (Ajzen, 1991; Esfandiar et al., 2019). 
$\mathrm{H} 2$ - El control percibido interno de la estudiante tiene un efecto positivo en la IE como carrera al finalizar sus estudios.

La formación universitaria impacta positivamente sobre la IE y la creación de negocios (Krueger et al. 2000)

H3- El contexto universitario, que fomente el emprendimiento tiene un efecto positivo en la actitud personal.

H4- El contexto universitario, que fomente el emprendimiento tiene un efecto positivo en el control interno percibido.

Para convertirse en una nación más innovadora, se debe trabajar sobre la cultura emprendedora a todo nivel de la sociedad para que las nuevas generaciones se desarrollen en liderazgo, creatividad, responsabilidad y ambición, y utilizar los beneficios de la educación universitarias femenina a pesar de los valores culturales y religiosos preexistentes (Van Ewijk \& Belghiti-Mahut, 2019)

H5- El contexto sociocultural, que fomente el emprendimiento tiene un efecto positivo en la actitud personal.

H6- El contexto sociocultural, que fomente el emprendimiento tiene un efecto positivo en el control interno percibido.

El efecto de tener familiares emprendedores cercanos y exitosos, son fundamentales por su inspiración para emprender su propio negocio al finalizar la carrera. (Criaco et al, 2017; Nowinski \& Haddoud, 2019; Krueger et al., 2000)

H7- El contexto familiar tiene un efecto positivo en la actitud personal.

H8- El contexto familiar tiene un efecto positivo el control percibido interno.

\section{Metodología}

El análisis se realiza en base a los datos recolectados en la octava edición del proyecto de investigación Global University Entrepreneurial Spirit Students' Survey (GUESSS, 2018), realizada de setiembre a diciembre 2018; en la que participaron 54 países y 3.000 universidades de todo el mundo. En este caso se utilizaron los datos de Uruguay.

Basado en la TBP (Ajzen, 1991), utilizada como base para determinar la intención de emprender como elección de carrera, influenciado por predisposición al comportamiento, normas subjetivas y su control percibido de la conducta. Las preguntas utilizadas están basadas en (Liñán \& Chen, 2009), a través de preguntas de escala Likert con 7 grados de acuerdo y desacuerdo, validados por varios estudios del equipo Guesss (Sieger, Fueglistaller, \& Zellweger, 2014).

Se ajustó un modelo de ecuaciones estructurales basado en covarianzas (CB-SEM) para la prueba de las hipótesis de investigación. Previamente al análisis de estimación del modelo, se calculó el coeficiente de Mardia (Mardia, 1970), para asegurar la presencia de normalidad multivariada en los datos obtenidos. No fue necesario realizar un
Análisis Factorial Exploratorio (EFA), al poseer resultados anteriores sobre la estructura del constructo (Krauss, Bonomo, \& Volfovicz, 2018 (b)).

Se efectuó un Análisis Factorial Confirmatorio (CFA) para probar si la estructura factorial exploratoria presenta índices de ajuste adecuados. Se utilizó el procedimiento de máxima verosimilitud para la evaluación de los modelos. La bondad de ajuste del modelo se probó utilizando el contraste Chi-cuadrado $\left(\chi^{2}\right)$, el cociente Chi-cuadrado/ grados de libertad del modelo ( $\left.\chi^{2} / g 1\right)$, la raíz del error cuadrático medio de aproximación (RMSEA), la raíz del error cuadrático medio residual (SRMR), el índice de ajuste comparativo (CFI), el índice de bondad de ajuste (GFI), el índice de bondad de ajustado (AGFI) y el índice de ajuste normalizado de Bentler-Bonett (NFI), (Byrne, 2001).

El análisis estadístico de los datos se realizó mediante el uso de los paquetes SPSS V.25 (IBM Corp., 2017) y AMOS V.23 (Abuckle, 2014)

\section{Desarrollo de la Investigación}

Se aplica un cuestionario voluntario en estudiantes de universidades públicas y privadas. Los estudiantes que contestaron la encuesta de forma válida en 2018 fueron 507 de los cuales, 275 eran mujeres correspondiendo a un $54 \%$ y siendo un $67 \%$ de ellas que provienen de las carreras en área empresarial y $94 \%$ eran estudiantes de grado. El $48 \%$ de las mujeres entrevistadas trabajan actualmente.

La validez y fiabilidad de las escalas se realizó mediante un análisis factorial confirmatorio (CFA). Se estimó el modelo a través de un diseño factorial con 6 variables y 32 indicadores, verificando los requisitos para este tipo de análisis, la existencia de al menos tres indicadores por factor. Asimismo, el tamaño de lo muestra $(n=275)$ supera los requisitos establecidos por diversos autores según los cuales el número mínimo estaría en 200 sujetos cuando el número de ítems es de 3 por factor o variable latente (Anderson \& Gerbing, 1984). Los parámetros fueron estimados mediante el procedimiento de máxima verosimilitud, al cumplirse los dos supuestos de normalidad. El supuesto de normalidad univariada puede afirmarse dado que los índices de asimetría y curtosis de los indicadores estuvieron por debajo del valor 2, (Bollen \& Long, 1993). De acuerdo a Bollen (1989) existe normalidad multivariada si el coeficiente de Mardia es menor a $\mathrm{p}^{\star}(\mathrm{p}+2)$, donde $\mathrm{p}$ es el número de variables observadas. En nuestro estudio existían 32 variables observadas (indicadores) y el coeficiente de Mardia provisto por el programa AMOS fue igual a 241,5, se puede afirmar la existencia de normalidad multivariada ya que el valor aportado por el programa es menor que el producto $32(32+2)=1088$. La presencia de normalidad en los datos confirma la conveniencia en la elección de este método.

La Tabla 1 presenta las cargas factoriales de cada uno de los indicadores, mostrando en todos los casos valores superiores a 0,70 . Se reporta además la validez del instrumento mediante el Alfa de Cronbach, indicando valores mayores a 0,80 , corroborándose la fiabilidad o consistencia interna de cada escala y por tanto la validez del constructo en la muestra de investigación. 
En la misma, las medidas de ajuste global aportan suficiente evidencia para poder aceptar el modelo de medición propuesto: el cociente entre los grados de libertad del modelo 2,750 es menor a cinco y se encuentra por tanto dentro del nivel recomendado
(Hair, Anderson, Tatham, \& Black, 1998). El valor obtenido de RMSEA $(0,080)$ y los niveles del GFI $(0,916)$, CFI $(0,928)$, NFI $(0,927)$ y AGFI $(0,837)$ suponen un nivel aceptable de confiabilidad y validez del modelo.

Tabla 1. Análisis factorial Confirmatorio. Fuente: Elaboración propia

\begin{tabular}{|c|c|c|}
\hline VARIABLES & $\begin{array}{c}\text { Carga } \\
\text { Factorial }\end{array}$ & Fiabilidad \\
\hline \multicolumn{3}{|l|}{ Contexto Universitario (CU) } \\
\hline El entorno de mi Universidad me inspira a desarrollar ideas para nuevos negocios. & 0,760 & \multirow{8}{*}{0,922} \\
\hline En mi Universidad, existe un clima favorable para convertirme en emprendedor. & 0,809 & \\
\hline En mi Universidad, los estudiantes son motivados para involucrarse en actividades emprendedoras. & 0,748 & \\
\hline $\begin{array}{l}\text { Los cursos y servicios a los que asistí...aumentaron mi entendimiento de las actitudes, valores y } \\
\text { motivaciones de los emprendedores. }\end{array}$ & 0,876 & \\
\hline $\begin{array}{l}\text { Los cursos y servicios a los que asistí......aumentaron mi entendimiento de las acciones que una } \\
\text { persona tiene que llevar a cabo para iniciar un negocio. }\end{array}$ & 0,830 & \\
\hline $\begin{array}{l}\text { Los cursos y servicios a los que asistí......aumentaron mis habilidades prácticas administrativas/de } \\
\text { gestión para poder iniciar un negocio. }\end{array}$ & 0,813 & \\
\hline Los cursos y servicios a los que asistí......aumentaron mi habilidad para desarrollar redes. & 0,822 & \\
\hline Los cursos y servicios a los que asistí......aumentaron mi habilidad para identificar una oportunidad. & 0,818 & \\
\hline \multicolumn{3}{|l|}{ Contexto Familiar (CF) } \\
\hline Reacción: Tu familia cercana. & 0,814 & \multirow{3}{*}{0,804} \\
\hline Reacción: Tus amigos. & 0,887 & \\
\hline Reacción: Tus compañeros de estudios. & 0,826 & \\
\hline \multicolumn{3}{|l|}{ Contexto Socio Cultural (CSC) } \\
\hline $\begin{array}{l}\text { En mi sociedad,... la influencia de una persona esta basado primariamene en: Habilidad de contribuir a } \\
\text { la sociedad/ Autoridad de la persona }\end{array}$ & 0,791 & \multirow{3}{*}{0,801} \\
\hline $\begin{array}{l}\text { En mi sociedad... se espera que los seguidores se espera que: Cuestionen a sus lideres cuando estan } \\
\text { en desacuerdo/ Obedezcan a los lideres sin cuestionamientos }\end{array}$ & 0,780 & \\
\hline $\begin{array}{l}\text { En mi sociedad, el poder es compartido en todos los niveles sociales/Concentrado en los valores } \\
\text { inferiores }\end{array}$ & 0,767 & \\
\hline \multicolumn{3}{|l|}{ Actitud Personal (AP) } \\
\hline Ser emprendedor implica más ventajas que desventajas para mí. & 0,718 & \multirow{5}{*}{0,916} \\
\hline Una carrera profesional como emprendedor me resulta atractiva. & 0,895 & \\
\hline Si tuviera la oportunidad y recursos, sería emprendedor. & 0,863 & \\
\hline Ser emprendedor implicaría grandes satisfacciones para mí. & 0,911 & \\
\hline Entre varias opciones, preferiría ser emprendedor. & 0,927 & \\
\hline \multicolumn{3}{|l|}{ Control Interno Percibido (CIP) } \\
\hline Identificar nuevas oportunidades de negocio. & 0,767 & \multirow{7}{*}{0,899} \\
\hline Crear nuevos productos y servicios. & 0,741 & \\
\hline Gestionar la innovación dentro de un negocio. & 0,803 & \\
\hline Ser un líder y comunicador. & 0,719 & \\
\hline Crear una red de contactos profesionales. & 0,838 & \\
\hline Comercializar una nueva idea o desarrollarla. & 0,862 & \\
\hline Administrar/Gestionar exitosamente un negocio. & 0,787 & \\
\hline \multicolumn{3}{|l|}{ Intención Emprendedora (IE) } \\
\hline Estoy dispuesto(a) a hacer cualquier cosa para ser un emprendedor. & 0,584 & \multirow{6}{*}{0,945} \\
\hline Mi meta profesional es llegar a ser emprendedor(a). & 0,814 & \\
\hline Haré todo lo posible para crear y gestionar mi propio negocio. & 0,829 & \\
\hline Estoy decidido(a) a crear una empresa en el futuro. & 0,872 & \\
\hline He pensado muy seriamente en iniciar un negocio. & 0,762 & \\
\hline Tengo la firme intención de crear un negocio algún día. & 0,850 & \\
\hline
\end{tabular}

ISSN: 0718-2724. (http://jotmi.org) 


\section{Resultados}

Modelo de ecuación estructural: contraste de las hipótesis del estudio comprobada la fiabilidad y validez de las escalas, pasamos a realizar el contraste de las hipótesis de investigación $\mathrm{H} 1$ a H8, así como a evaluar la bondad de ajuste del modelo estructural.

Figura 2. Resultado del modelo de ecuaciones estructurales (se presentan los coeficientes de regresión betas estandarizados y sus respectivas significaciones).

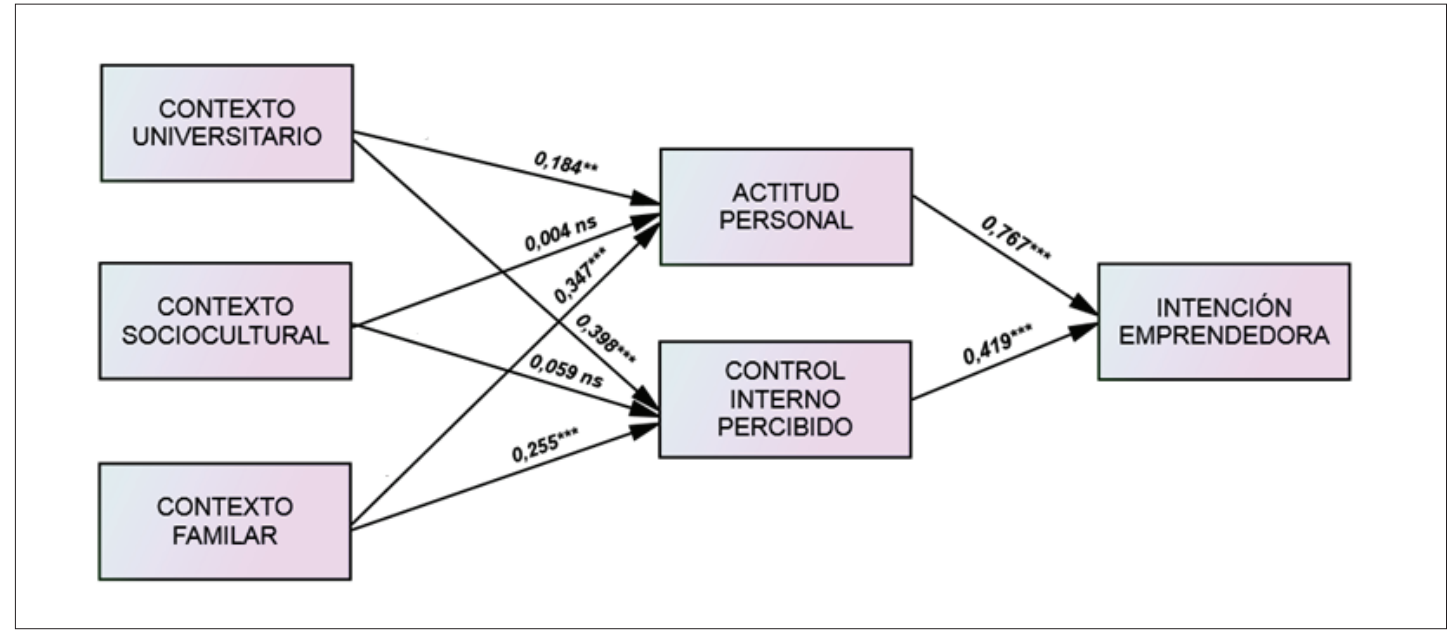

Fuente: Elaboración propia

La figura 2 presenta los resultados del modelo de ecuación estructural para la muestra de estudiantes universitarias. En la Tabla 2 presenta los valores de los coeficientes betas estandarizados del modelo, así como sus respectivos errores estándares (S.E.), razones críticas (C.R.) y significaciones (P). Los coeficientes del modelo estructural que manifiestan la relación existente entre las variables permiten probar afirmativamente las hipótesis $\mathrm{H} 1, \mathrm{H} 2, \mathrm{H} 3, \mathrm{H} 4, \mathrm{H} 7$ y $\mathrm{H} 8$ (todas con valores de significación menor a 0,01), y rechazar las hipótesis H5 y H6 (Tabla 2 y Tabla 3). Es así como se puede afirmar que la Actitud Personal (H1; beta estandarizado $b=0,767 ; \mathrm{p}<0,001$ ) y el Control Interno Percibido $(\mathrm{H} 2 ; \mathrm{b}=0,419 ; \mathrm{p}<0,001)$ tienen un impacto positivo $\mathrm{y}$ significativo sobre la IE (Tabla 2).

Tabla 2. Resultados del modelo de ecuación estructural (S.E.: error estándar; C.R.: razón crítica; p: valores p y significación).

\begin{tabular}{|ccc|c|c|c|c|}
\hline \multicolumn{2}{|c|}{ Relación entre Variables Latentes } & Estimador & S.E. & C.R. & P \\
\hline Actitud Personal & $\leftarrow$ & Contexto Universitario & 0.184 & 0.056 & 3.287 & 0.001 \\
Control Interno Percibido & $\leftarrow$ & Contexto Universitario & 0.398 & 0.053 & 7.508 & $* * *$ \\
Actitud Personal & $\leftarrow$ & Contexto Familiar & 0.347 & 0.056 & 6.194 & $* * *$ \\
Control Interno Percibido & $\leftarrow$ & Contexto Familiar & 0.255 & 0.053 & 4.812 & $* * *$ \\
Actitud Personal & $\leftarrow$ Contexto Socio Cultural & 0.004 & 0.055 & 0.066 & $0.947 \mathrm{~ns}$ \\
Control Interno Percibido & $\leftarrow$ Contexto Socio Cultural & 0.059 & 0.052 & 1.15 & $0.25 \mathrm{~ns}$ \\
Intención Emprendedora & $\leftarrow$ & Actitud Personal & 0.731 & 0.034 & 21.217 & $* * *$ \\
Intención Emprendedora & $\leftarrow$ Control Interno Percibidd & 0.142 & 0.034 & 4.133 & $* * *$ \\
\hline
\end{tabular}

Del mismo modo se puede afirmar que tanto el Contexto Universitario (H3; $b=0,184 ; \mathrm{p}=0,001$ y H4; $b=0,398 ; \mathrm{p}<0,001)$ como el Contexto Familiar (H7; $b=0,347 ; p<0,001$ y H8; $b=0,255 ; p<0,001)$ tienen una influencia positiva significativa sobre las variables: Actitud Personal y Control Interno Percibido (Tabla 2). Por otra parte, el Contexto Socio Cultural no tiene un efecto significativo sobre la Actitud Personal y el Control Interno Percibido (H5; b=0,004; $\mathrm{p}=0,947$ y H6; b=0,059; $\mathrm{p}=0,25$ respectivamente).
Al comparar la magnitud de los coeficientes beta, se observa que la Actitud Personal fue la variable que tuvo mayor impacto sobre la IE $(0,767)$, seguida por el Control Interno Percibido $(0,419)$. El Contexto Universitario y el Contexto Familiar afectaron indirectamente a la Intención Emprendedora a través del cambio en la Actitud Personal y el Control Interno Percibido (Tabla 2). 
Tabla 3. Conclusiones del contraste de Hipótesis. Fuente: Elaboración propia

\begin{tabular}{|c|c|c|c|}
\hline Hipótesis & Variable Independiente & Variable Dependiente & Conclusión \\
\hline H1 & Actitud Personal & Intención Emprendedora & Soportada \\
H2 & Control Interno Percibido & Intención Emprendedora & Soportada \\
H3 & Contexto Universitario & Actitud Personal & Soportada \\
H4 & Contexto Universitario & Control Interno Percibido & Soportada \\
H5 & Contexto Socio Cultural & Actitud Personal & No soportada \\
H6 & Contexto Socio Cultural & Control Interno Percibido & No soportada \\
H7 & Contexto Familiar & Actitud Personal & Soportada \\
H8 & Contexto Familiar & Control Interno Percibido & Soportada \\
\hline
\end{tabular}

El ajuste global del modelo estructural se probó mediante el cociente $\chi^{2} / g l$, el RMSEA, y los índices GFI, CFI, NFI y AGFI. Se puede afirmar que los resultados del ensayo del modelo indicaron un ajuste aceptable (Tabla 4). El valor del cociente $\chi^{2} / g l,(3,56)$ se encuentra dentro del intervalo de aceptación del modelo $(<5)$, (Hair, Anderson, Tatham, \& Black, 1998). Los índices de bondad de ajuste del modelo RMSEA $(0,088<0,09)$, así como GFI $(0,903)$, CFI $(0,910)$ y NFI $(0,917)$ resultaron adecuados (superiores a 0,90$)$, AGFI $(0,816)$ fue el único índice que presento un valor por debajo del aceptable $(0,90)$, siguiendo los valores de referencia de Arbuckle (2007).
Tabla 4. Índices de bondad de ajuste del modelo estructural.

\begin{tabular}{|c|c|c|c|c|c|}
\hline$\chi^{2} / g l$ & RMSA & GFI & CFI & NFI & AGFI \\
\hline 3,56 & 0,088 & 0,903 & 0,91 & 0,917 & 0,816 \\
\hline
\end{tabular}

Fuente: Elaboración propia

Se muestra la matriz de correlaciones (coeficientes de correlación lineal de Pearson) entre las variables en la Tabla 5; se observa que todas las correlaciones están por debajo de 0,40 , sugiriendo la independencia entre las variables.

Tabla 5. Coeficientes de correlación lineal de Pearson(r) entre las variables. Fuente: Elaboración propia

\begin{tabular}{|l|ccccc|}
\hline & $\begin{array}{c}\text { Contexto } \\
\text { Universitario }\end{array}$ & $\begin{array}{c}\text { Contexto } \\
\text { Familiar }\end{array}$ & $\begin{array}{c}\text { Contexto } \\
\text { Socio Cultural }\end{array}$ & $\begin{array}{c}\text { Actitud } \\
\text { Personal }\end{array}$ & $\begin{array}{c}\text { Control } \\
\text { Interno } \\
\text { Percibido }\end{array}$ \\
\hline Contexto Universitario & 1 & $0,219 * *$ & $-0,032 \mathrm{~ns}$ & $0,260 * *$ & $0,352 * *$ \\
Contexto Familiar & & 1 & $-0,036 \mathrm{~ns}$ & $0,387 * *$ & $0,340 * *$ \\
Contexto Socio Cultural & & & 1 & $-0,015 \mathrm{~ns}$ & $0,038 \mathrm{~ns}$ \\
Actitud Personal & & & & 1 & $0,339 * *$ \\
Control Interno Percibido & & & & 1 \\
\hline
\end{tabular}

${ }^{\star *}$ La correlación es significativa al nivel 0,01 (bilateral), ns= no significativa

\section{Discusión}

Este estudio busca ser un punto de partida para impulsar el proceso de la educación emprendedora, fortaleciendo aquellos factores que impactan sobre la Intención emprendedora como parte del empoderamiento de las universitarias (Bae, Qian, Miao, \& Fiet, 2014; Marín Rives et al., 2015).

Se analizó a través de un modelo de ecuaciones estructurales las relaciones entre tres tipos de Contextos (Universitario, Sociocultural y Familiar) y su efecto sobre la Intención Emprendedora. Los resultados obtenidos muestran que el Contexto Universitario y el Contexto Familiar tuvieron efectos positivos significativos en las variables Actitud Personal y el Control Percibido Interno. El Contexto Universitario y el Contexto Familiar afectaron indirectamente a la Intención Emprendedora a través del cambio en la Actitud Personal y el Control Interno Percibido.

En el caso del Contexto Socio Cultural, no se encontró un efecto significativo sobre la Actitud Personal y el Control Percibido Interno, de esta forma se ratifica que el contexto sociocultural no impacta en la
IE como se vio en un estudio previo del GUESSS 2016 en estudiantes universitarias en Uruguay (Krauss et al., 2018b). En particular una posible explicación para este resultado en Uruguay puede provenir de dos condiciones, por un lado, la poca valoración del rol del emprendedor y por otro, la seguridad que brinda tener un empleo público. A nivel histórico, el sueño de todo ciudadano es trabajar en el Estado, al punto que en 2016, se presentaron 398.553 personas para 1.092 cargos, según datos del Sistema de Reclutamiento y Selección de Uruguay Concursa en un relevamiento que realizó la Oficina Nacional del Servicio Civil (ONSC) en el Informe y Memoria Anual de la Gestión del Gobierno Nacional (González Keusseian, 2018).

En el marco del Sistema Nacional de Transformación Productiva y Competitividad (Uruguay Transforma) que promueve el emprendimiento en el país, el Estado busca fortalecer el ecosistema a través de diferentes propuestas brindando herramientas, sensibilización, formación, en todo el país a través del Portal Emprendedor (https://uruguayemprendedor.uy/).

Además, en un hecho histórico se presenta y aprueba en 2019, la Ley de Fomento del Emprendedurismo que contiene tres capítulos, el 
primero define y fomenta la cultura emprendedora en edades tempranas, el segundo crea la Sociedad por Acción Simplificada (SAS) que facilita la formalización y la incorporación de inversores en startup y el tercero habla del financiamiento colectivo (crowfunding) que todavía debe ser articulado con el Banco Central del Uruguay (Uruguay Presidencia, 2019).

Es importante que las Universidades aprovechen estas oportunidades y potenciarlas a través de su enseñanza emprendedora, el trabajo de sus incubadoras, para impulsar el cambio cultural hacia la valoración del rol del emprendedor.

En nuestra investigación, se muestra evidencia empírica que la Actitud Personal tuvo mayor impacto que el Control Interno Percibido sobre la Intención de Emprender.

Otro de los resultados del estudio es que fortifica la validez de la TPB como fundamento para explicar la IE, particularmente en el caso de mujeres universitarias ya que la variable que tiene mayor impacto sobre la IE es la Actitud Personal de las universitarias.

A nivel de las estrategias pedagógicas, la educación emprendedora puede desarrollar la habilidad individual a través de la metodología de resolución de problemas, generando un reconocimiento de oportunidades para emprender y un comportamiento innovador que se potencia con las diferentes formas ágiles de enseñanza: design thinking, team working, business startups, visitar proyectos exitosos, ejercicios basados en resolución de problemas y reuniones con emprendedores (Welsh, Tullar, \& Nemati, 2016; Ji Young, Dae Soo, Chang-Soo, \& Park, 2018; Esfandiar et al., 2019).

El propiciar ambientes que promuevan una mentalidad empresarial, el aprender emprendiendo (Neck, Greene, \& Brush, 2014; Din, Anuar, \& Usman, 2016), con un enfoque muy centrado en el desarrollo de las competencias emprendedoras y vivir la experiencia de llevar a cabo un emprendimiento real e innovador, con profesores y mentores involucrados en el desarrollo de competencias que favorece el impulso a ser emprendedores nacientes (Ruskovaara, Hämäläinen, \& Pihkala, 2016; Nowinski \& Haddoud, 2019). Las mismas habilidades emprendedoras desarrolladas a nivel universitario, se ha probado que genera un impacto mayor en el desarrollo económico de los países si se inicia a nivel de educación terciaria o en niveles anteriores (Pandit, Joshi, \& Tiwari, 2018). Si bien los resultados son variables, el comenzar en estadios tempranos de la educación es importante.

La llave para promover la IE que ha probado ser más efectiva y que debe tenerse en cuenta en todo proceso de enseñanza, sería un conjunto de actividades de EE (Educación Emprendedora) que las empoderen a través de la autorrealización (motivación pull), generar roles inspiradores como modelos, una actitud positiva hacia el emprendedurismo y autoeficacia emprendedora (Nowinski \& Haddoud, 2019; Özsungur, 2019).

En Uruguay, una experiencia previa en la Universidad Católica analizó el efecto ex ante y ex post de un curso de Emprendedurismo e Innovación en todas las carreras de la Facultad de Ciencias
Empresariales, en donde los estudiantes debían de realizar un emprendimiento real en equipo y obtener ventas. Se midieron las competencias emprendedoras en un proceso de EE mostrando un incremento en las actitudes, motivaciones e intenciones de todos los estudiantes para emprender luego de la experiencia; se destaca que en las estudiantes mujeres se constató un aumento importante en la competencia de persuasión y creación de redes de apoyo que era un factor débil en ellas. (Krauss, Bonomo, \& Volfovicz, 2018a).

En el caso particular de las estudiantes universitarias, para empoderarlas y que respondan a los desafíos de la 4ta Revolución, se deberá el impulsar un fortalecimiento en su creatividad, autoeficacia, iniciativa, autoconfianza, autoestima y su capacidad de emprender, mediante la realización un pool de cursos y talleres que les permitan experimentar el realizar un emprendimiento, fortalecer sus capacidades creativas, de liderazgo y de resolución práctica de problemas.

Asimismo, será necesario que tengan charlas y se vinculen con mujeres emprendedoras que sean exitosas, especialmente si tienen edades similares, lo que las hace identificarse con ellas y ver que es posible emprender y tener un equilibrio entre su emprendimiento y su propia familia, que es uno de los desafíos de la educación 4.0.

El estudio tiene la limitación de no haberse realizado a todos los estudiantes de las Universidades (por ser de respuesta voluntaria) provocando la baja tasa de respuesta y tener la mayor proporción de estudiantes de grado empresariales. A su vez, como futuras investigaciones, sería interesante realizar estudios comparativos a nivel Latinoamericano y mundial para detectar similitudes o diferencias en los comportamientos.

\section{Referencias}

Ajzen, I. (1991). Theory of Planned Behavior. Organization Behavior and Human Decision Processes, 50(2), 179-211. doi:10.1016/j. obhdp.2010.11.001

Anderson, J. C., \& Gerbing, D. W. (1984). The effect of sampling error on convergence, improper solutions, and goodness-of-fit indices for maximum likelihood confirmatory factor analysis. Psychometrika, 49, 155-173. doi:10.1007/BF02294170

Arbuckle, J.L. (2007). Amos 16.0 User's Guide. Chicago, IL: SPSS Inc

Asante, E. A., \& Affum-Osei, E. (2019). Entrepreneurhip as a career choice: The impact of locus of control on aspiring entreprenerurs' opportunity recognition. Journal of Business Research, 98, 227-235. doi:10-1016/j.jbusres.2019.02.006

Azis, M., Maulana Haeruddin, M. I., \& Azis, F. (2018). Entreprenuership Education and Career Intention: The Perks of being a Woman Student. Journal of Entrepreneurship Education, 21(1), 1-10

Bae, T., Qian, S., Miao, C., \& Fiet, J. (2014). The relationshipp between EE and EI: a meta-analytic review. Entrepreneurship Theory and Practice, 38(2), 217-254. doi:10.1111/etap.12095 
Bogatyreva, K., Edelman, L., Manolova, T., \& Osiyevskyy, O. (2019). When do entrepreneurial intentions lead to actions? The role of national culture. Journal of Business Research(96), 309-321. doi:10.1016/j. jbusres.2018.11.034

Bollen, K. A. (1989). Structural equations with latent variables. New York: John Wiley y Sons. doi:10.1002/978111861979

Bollen, K. A., \& Long, J. S. (1993). Testing Structural Equation Models. Newbury Park, CA: Sage

Byrne, B. M. (2001). Structural equation modeling with AMOS. Basic concepts, applications, and programming. Londres: LEA.]

Criaco, G., Sieger, P., Wennberg, K., Chirico, F., \& Minola, T. (2017). Parents' performance in entrepreneurship as a "double-edged sword" for the intergenerational transmission of entrepreneurship. Small Business Economics, 49(4), 1-24. doi:10.1007/s11187-017-9854-x

Din, B. H., Anuar, A. R., \& Usman, M. (2016). The Effectiveness of the Entrepreneuship Education Program in Upgrading Entrepreneurial Skills among Public University Students. Procedia Social and Behavioral Sciences, 224, 117-123.

Esfandiar, K., Sharifi-Tehrani, M., Pratt, S., \& Altinay, L. (2019). Understanding entrepreneurial intentions: A developed integrated structural model approach. Journal of Business Research, 172-182. doi:10.106/j.jbusres.2017.10.045

Fayolle, A., \& Liñan, F. (2014). The future of research on entrepreneurial intention. Journal of Business Research, 67(5), 663-666. doi:10.1016/j.jbusres.2013.11.024

GEM. (2016/2017). Women's Entrepreneurship Report. Global Entrepreneurship Research Association.

González Keusseian, N., (2018). Postulaciones para trabajar en el Estado cayeron a la mitad. [En línea] Availableat: https://www.elpais.com.uy/informacion/postulacionescayeron-mitad.html Recuperado 10072020.

GUESSS. (2018). Guesssurvey.org. Retrieved 4 25, 2019, from Datacollections: http://www.guesssurvey.org/datacollections/

Hair, J., Anderson, R., Tatham, R., \& Black, W. (1998). Multivariate data analysis (5th ed.). New York, USA: Prentice Hall College Div.

Ji Young, K., Dae Soo, C., Chang-Soo, S., \& Park, J. Y. (2018). The role of problem solving ability on innovative behavior and opportunity recognition in university students. Journal of Open Innovation, 4(1), 1-13. doi:10.1186/s40852-018-0085-4

Joensuu, A., Viljamaa, A., \& Varamaki, E. (2013). Development of entrepreneurial intention in higher education and the effect of gender- a latent growth curve analysis. Education + Training, 55(8/9), 781-803. doi:10.1108/ET-06-2013-0084
Krauss, C., Bonomo, A., \& Volfovicz, R. (2018a). La educación emprendedora frente al impacto de la 4ta Revolución Industrial. Teuken Bidikay, 9(12), 193-217. doi:10.33571/teuken

Krauss, C., Bonomo, A., \& Volfovicz, R. (2018b). La intención emprendedora femenina en las estudiantes universitarias de Uruguay. 53 asamblea Anual de CLADEA, San José, Costa Rica. Memorias LIII Asamblea Anual CLADEA 2018 http://www.cladea.org/proceeding-2018/pdf/papers/Emprendimiento/CLADEA_2018_paper_235.pdf. ISBN 978-612-46367-7-6

Krueger, N. F., Reilly, M. D., \& Carsrud, A. L. (2000). Competing Models of Entrepreneurial Intentions. Journal of Business Venturing, 15, 411-432. doi:10.1016/S0883-9026(98)00033-0

Liñán, F., \& Chen, Y. (2009). Development and cross-cultural application of a specific instrument to measure entrepreneurial intentions. Entrepreneurship: Theory And Practice, 33(3), 593-617.

Mardia, K. V. (1970) "Measures of multivariate skewness and kurtosis with applications”, Biometrika 57, pp. 519-530

Marín Rives, L., Rubio Bañón, A., \& Sánchez-Mora, M. I. (2015). El impacto del entorno del estudiante en sus intenciones de crear una empresa cuando finalice sus estudios. Lan Harremanak, 32(1), 504-526.

Mckinsey Global Institute. (2015). How advancing women's equality can add \$12 Trillon to global growth. Retrieved 4 2019, from McKinsey Global Institute: www.mckinsey.com

MIEM, 2017. Encuesta nacional de mipymes industriales, comerciales $y$ de servicio. [En línea] Available.at: https://observatorio.miem.gub. uy/obs/sites/default/files/documentos/encuesta_nacional_de_mipymes_2017_0.pdf [Último acceso: 2011 2019].

Naranjo, C. (2019). Desigualdades de género en el emprendimiento y en los negocios de las mujeres. Revista Trabajo Social, (86), 3-12. Consultado de http://redae.uc.cl/index.php/RTS/article/view/2574

Neck, H. M., Greene, P. G., \& Brush, C. G. (2014). Teaching Entreprenuership. A Practice-Based Approach. Cheltenham: Edward Elgar Publishing Limited.

Newbery, R., Lean, J., Moizer, J., \& Haddoud, M. (2018). Entrepreneurial identity formation during the initial entrepreneurial experience: The influence of simulation feedback and existing identity. Journal of Business Research, 85, 51-59. doi:10.1016/j.jbusres.2017.12.013

Nicolaou, N., Shane, S., Cherkas, L., \& Spector, T. (2009). Opportunity recognition and the tendency to be an entrepreneur: A bivariate genetics perspective. Organizational Behavior and Human Decision Process, $110(2), 108-117$.

Nowinski, W., \& Haddoud, M. Y. (2019). The role of inspiring role models in enhancing entrepreneurial intention. Journal of Business Research, 96, 183-193. doi:10.1016/j.jbusres.2018.11.005 
Ortiz García, P., \& Olaz Capitán, Á. (2018). Factores competenciales en el emprendimiento de la mujer. Revista Española de Sociología , 27(3), 413-432. doi:10.22325/fes/res.2018.21

Özsungur, F. (2019). A research on women`s entrepreneurship motivation: Sample of Adana Province. Women's Studies International Forum, 74, 114-126. doi:10.1016/j.wsif.2019.03.006

Pandit, D., Joshi, M. P., \& Tiwari, S. R. (2018). Examining Entrepreneurial Intention in Higher Education: An Exploratoy Study of College Students in India. The Journal of Entrepreneurship, 27(1), 25-46. doi:10.1177/0971355717738595

Roomi, M., Rehman, S., \& Henry, C. (2018). Exploring the normative context for women's entrepreneurship in Pakistan: a critical analysis. International Journal of Gender and Entrepreneurship, 10(2), 158-180. doi:10.1108/IJGE-03-2018-0019

Ruskovaara, E., Hämäläinen, M., \& Pihkala, T. (2016). Head teachers managing entrepreneurship education- Empirical evidence from general education. Teaching and Teach education, 55, 155-164. doi:10.1016/j.tate.2016.01.004

Sieger, P., Fueglistaller, U., \& Zellweger, T. (2014). Student Entrepreneurship Across the Globe: A Look at Intentions and Activities. St.Gallen: KMU-HSG.

Stevenson, L. (1986). Against all odds: The entrepreneurhip of women. Journal of Small Business Management, 24(4), 30-36.
Uruguay Presidencia (2019). El Parlamento aprobó ley que facilita la creación de nuevas empresas y su financiamiento. [En línea] Available at: https://www.presidencia.gub.uy/comunicacion/comunicacionnoticias/martin-dibarbure-ande-ley-fomento-emprendedurismo-parlamento [Último acceso: 1007 2020].

Van Ewijk, A. R., \& Belghiti-Mahut, S. (2019). Context, gener and entrepreneurial education changes the equation. International Journal of Gender and Entrepreneurship, 11(1), 75-98. doi:10.1108/IJGE-052018-0054

Van Gelderen, M., Kautonen, T., \& Fink, M. (2015). From entreprenuerial intentions to actions: Self-control and action- related doubt, fear, and aversion. Journal of Business Venturing, 30, 655-673. doi:10.1016/j.jbusvent.2015.01.003

Veiga, L., Chaquiriand, I., \& Bartesaghi, A. (2017, Febrero). Las barreras a la actividad emprendedora en Uruguay. Revista de Negocios del IEEM, 20(1), 48-54.

Welsh, D. H., Tullar, W. L., \& Nemati, H. R. (2016). Entrepreneurship education: Process, method, or both? Journal of Innovation \& Knowledge, 1(3), 125-132. doi:10.1016/j.jik.2016.01.005

World Economics Forum. (2018). The Global Gender Gap Report. Cologny: World Economic Forum. Retrieved from www.weforum.org 
